\title{
THE INTENSITY DISTRIBUTION OF THE EMISSION BEAM AND A DISCUSSION OF THE RADIO LUMINOSITY OF PULSARS
}

\author{
XINJI WU \\ Geophysics Department, Peking University \\ R. N. MANCHESTER \\ Division of Radiophysics, CSIRO
}

\begin{abstract}
A new radio luminosity equation for pulsars is presented which is based on the geometry of a polarcap model. Statistical results show that the emission component intensity distribution is Gaussian. There are 100 pulsars with new radio luminosity values. We show that the radio luminosity can be described by one relation $L \propto\left(B P^{2}\right)^{1.07}$ or $L \propto Q^{\prime-1.4}, Q^{\prime}=2 P^{1.1} \dot{P}_{-15}^{-0.4}$ over the whole range of period and period derivatives, contrary to other recently suggested luminosity laws.
\end{abstract}

\section{Introduction}

Questions of current interest concern the radio luminosity law. It is generally believed that the luminosity depends on some basic physical properties of the neutron star. Gunn and Ostriker (1970) and Lyne et al. (1985) assumed that $L \propto B^{2}$. Luminosity laws of the form $L \propto P^{\alpha} \dot{P}^{\beta}$ have been given by some authors: $L \propto \dot{P} P^{-4}$ and $L \propto \dot{P} P^{-5}$ (Wu et al. $1980), L \propto P^{-0.86} \dot{P}^{0.38}$ (Vivekanand and Narayan $1981), L \propto P^{-1.04} \dot{P}^{0.35}$ (Prószyński and Przybycień 1984), $L \propto \dot{P} P^{-3}$ (Xie et al. 1985). In those investigations it is assumed that the same luminosity relation holds over the entire range of periods and period derivatives; that this may not be the case is suggested by a plot presented by Taylor $e t$ al. (1986), in which the luminosity of pulsars is plotted against the parameter $Q^{\prime}=2 P^{1.1} \dot{P}^{-0.4}$. It appears that $L$ is nearly independent of $Q^{\prime}<0.7$, but then decreases rather steeply as $Q^{\prime}$. A similar conclusion was obtained by Stollman (1987): $L$ cannot be described by one relation over the entire range of $B P^{-2}$ values; $L$ is best described by $L \propto B P^{-2}$ for $B P^{-2}<10^{13} \mathrm{G} \mathrm{s}^{-2}$ and $L \sim$ constant for $B P^{-2}>10^{13} \mathrm{G} \mathrm{s}^{-2}$. The most important difference is whether the luminosity relations hold over the entire range or not.

\section{The intensity distribution of the emission cone}

All pulsar emission profiles represent a dynamic superposition of distinct contributions from various emitting regions within the beam. This is the physical basis behind the concept of a component. In many cases, the sight line only sweeps over one individual emission range, or component, such as the conal single profile, core single profile and partial cone single profile. We assumed that the intensity distribution of every component of emission cone is a Gaussian distribution

$$
I=I_{0} e^{-A^{2} \rho^{2}},
$$

where $A$ is a constant, $\rho$ is the variable value indicating the width of a family of nested cones. There is a relation for the polar cap model (Manchester and Taylor 1977),

$$
\cos \rho=\cos (\alpha+\beta) \cos \alpha+\sin (\alpha+\beta) \sin \alpha \cos \Delta \varphi
$$

where $\alpha$ is magnetic inclination angle, $\beta$ is the angle between sight line and magnetic axis, and $\Delta \varphi$ is the half apparent beamwidth. When the values of $\rho, \beta$ and $\Delta \varphi$ are not very large (less than $57^{\circ}$; for most pulsars these conditions exist), then eq.(2) becomes

$$
\rho^{2}=\beta^{2}+\sin (\alpha+\beta) \sin \alpha(\Delta \varphi)^{2} .
$$

Eq.(1) can be rewritten as

$$
\begin{gathered}
I=I_{0} e^{-A^{2} \beta^{2}} e^{-A^{\prime 2}(\Delta \varphi)^{2}}=I^{\prime} e^{-A^{\prime 2}(\Delta \varphi)^{2}} \\
A^{\prime 2}=A^{2} \sin (\alpha+\beta) \sin \alpha .
\end{gathered}
$$

When $\beta=0$,

$$
I=I_{0} e^{-A^{2} \sin ^{2} \alpha\left(\Delta \varphi^{\prime}\right)^{2}},
$$

where $\Delta \varphi^{\prime}$ is the half apparent beamwidth. In the case of $\beta=0$ this means that the sight line sweeps the emission cone from the center.

For a Gaussian distribution, when the sight line sweeps the emission cone with angle $\beta$, the cross section is also a Gaussian distribution. This conclusion is important. It means that all of the mean pulse profiles of the individual emission components have similar distributions. From eq.(4) we obtained

$$
A^{\prime 2}=\frac{\ln 10}{\left(\Delta \varphi_{10}\right)^{2}}=\frac{\ln 2}{\left(\Delta \varphi_{50}\right)^{2}}=\frac{\pi / 4}{\left(\Delta \varphi_{\mathrm{e}}\right)^{2}},
$$




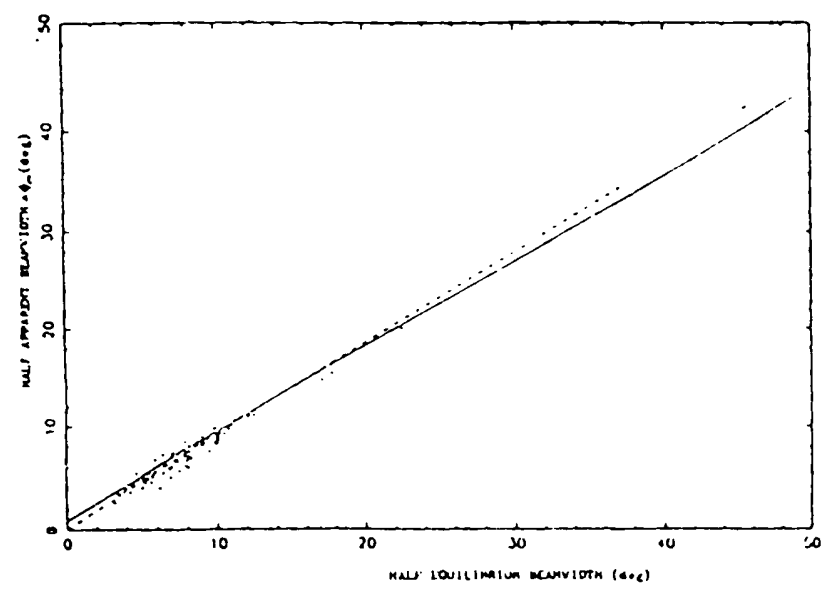

Figure $1 \Delta \varphi_{50}$ vs. $\Delta \varphi_{\mathrm{e}}$ for 92 pulsars with single profiles. The dashed line is the theoretical Gaussian and the solid line is the regression line.

where $\Delta \varphi_{10}$ and $\Delta \varphi_{50}$ are the half apparent beamwidth for $10 \%$ and $50 \%$ of peak value, and $\Delta \varphi_{\mathbf{e}}$ is the half equilibrium beamwidth. A useful relation is obtained from eq.( 7 ,

$$
\Delta \varphi_{50}=0.94 \Delta \varphi_{\mathrm{e}}=0.55 \Delta \varphi_{10} .
$$

The parameters of $\Delta \varphi_{50}, \Delta \varphi_{10}$ and $\Delta \varphi_{\mathrm{e}}$ are the observables. Eq.(8), derived from a Gaussian distribution, offers us a method to test whether the mean pulse profile is Gaussian. We should expect that the observational parameters $\left(\Delta \varphi_{50}, \Delta \varphi_{10}\right.$ and $\left.\Delta \varphi_{\mathrm{e}}\right)$ would be in agreement with eq. (8) for pulsars with a single mean pulse profile.

The statistical sample chosen from the paper of Lyne and Manchester (1988) is divided into two classes: (1) single profiles (92 pulsars), and (2) well resolved double profiles (21 pulsars). We discuss each component. The theoretical relations for the three apparent beamwidths mentioned above are the standards for testing. Linear regression equations and correlation coefficents have been calculated for the two classes of pulsars.

For the single-component class (92 pulsars) the linear regression results are

$$
\begin{array}{ll}
\Delta \varphi_{50}=0.87 \Delta \varphi_{\mathrm{e}}+0.42, & R=0.96 \\
\Delta \varphi_{10}=1.55 \Delta \varphi_{\mathrm{e}}+1.51, & R=0.96
\end{array}
$$

in good agreement with a Gaussian distribution.

For the well-resolved double class (42 single components), the results show that each single component is nearly Gaussian. The statistical results are

$$
\begin{aligned}
& \Delta \varphi_{50}=0.95 \Delta \varphi_{\mathrm{e}}-0.39, \quad R=0.99 \\
& \Delta \varphi_{10}=1.65 \Delta \varphi_{\mathrm{e}}+0.44, \quad R=0.97 .
\end{aligned}
$$

All of these correlation coefficents are much higher than the pass values at $0.1 \%$ level of significance.

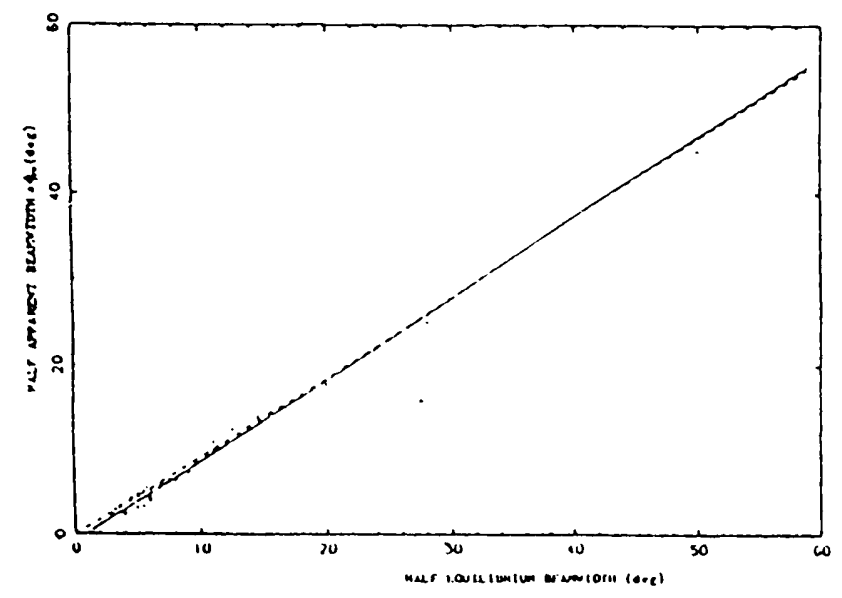

Figure $2 \Delta \varphi_{50}$ vs. $\Delta \varphi_{\mathrm{e}}$ for 42 single components of 21 pulsars; see figure 1 for key.

The pass values of $R$ are 0.34 and 0.49 for a sample size of 92 and 42 respectively. Figures 1 and 2 show the statistical and theoretical results. The regression line is near the theoretical Gaussian distribution.

\section{Discussion: radio luminosity formula}

The radio luminosity formula given by Taylor and Manchester (1975) is

$$
L_{1}=\pi^{3}\left(W_{\mathrm{e}} / P\right) d^{2} S_{400} \Delta f
$$

This simple formula has also been used in some research works, such as discussion of the radio luminosity laws (Taylor and Stinebring 1986, Stollman 1987).

$$
L_{2}=S_{400} d^{2}\left(\mathrm{mJy} \mathrm{kpc}^{2}\right),
$$

where the $d$ is th e ristance to pulsar, $S_{400}$ is the average flux density at $400 \mathrm{MHz}$, and $\Delta f$ is the frequency bandwidth. Eq.(9) is correct if $\beta=0$ and $\rho=\Delta \varphi_{e}$. Eq.(10) is equivalent to eq.(9) when the values of the pulse equivalent width are constant for any pulsar. In general, however, $\beta \neq 0, \rho \neq \Delta \varphi_{\mathrm{e}}$, and $\Delta \varphi_{e}$ is also not constant. For a long period after suggesting this formula we did not know how to estimate the values of $\alpha, \beta$ and $\rho$.

A discussion about a modified radio luminosity formula has been presented elsewhere (Wu et al. 1986). The conclusion is that the old formula must be corrected by two factors: one is a function of $Q$ only; another is the value of $\left(\rho / \Delta \varphi_{0}\right)^{2}$.

The problem is that the intensity distribution of emission is nearly Gaussian. So it is neccessary to restudy the new formula of radio luminosity. The relation $\rho=6.5 P^{-1 / 3}$ given by Lyne et al. (1988) 


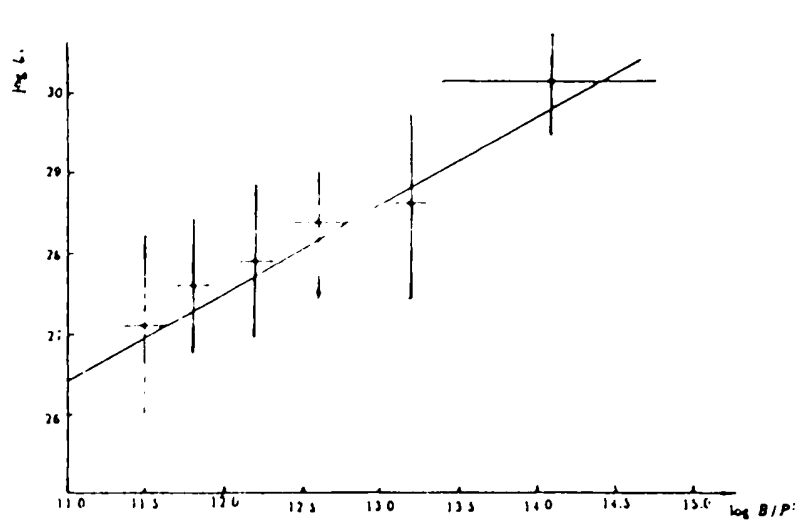

Figure $3 \log L_{3}$ vs. $\log B P^{-2}$ for 100 pulsars chosen from the catalog of Lyne and Manchester (1988). The luminosity, $L_{3}$, is defined by eq.(27). The line is given by the regression equation.

allows us to estimate the values of $\alpha, \beta, \rho$ and $Q$ using eq.(2) and following equations

$$
\begin{gathered}
\left(\frac{\partial \psi}{\partial \varphi}\right)_{\max }=\frac{\sin \alpha}{\sin \beta} \\
\rho=6.5 P^{-1 / 3},
\end{gathered}
$$

where $(\partial \psi / \partial \varphi)_{\max }$ is the maximum gradient of position angle.

Based the assumption that the emission beam intensity distribution is Gaussian, the flux density of the pulse received on earth is

$$
S_{400} P=2 \int_{0}^{\Delta \varphi} I^{\prime} \exp \left(-A^{\prime 2} x^{2}\right) \frac{P}{2 \pi} d x
$$

Combining eqs.(4), (5) and (7), eq.(13) becomes

$$
S_{400} P=I_{0} P \exp \left(-A^{2} \beta^{2}\right) \Delta \varphi_{\mathrm{e}} / \pi .
$$

In the case of $\beta=0$, where the sight line sweeps through the center of the emission cone, the flux density of pulse received on earth is

$$
\begin{gathered}
S_{400}^{\prime} P=I_{0} W_{\mathrm{e}}^{\prime} \\
S_{400}^{\prime}=\frac{\Delta \varphi_{\mathrm{e}}^{\prime}}{\Delta \varphi_{\mathrm{e}}} S_{400} \exp \left(A^{2} \beta^{2}\right),
\end{gathered}
$$

where $\Delta \varphi_{e}^{\prime}$ is the half apparent beamwidth in the case $\beta=0$. The new radio luminosity formula is obtained

$$
L_{3}=L_{1} K_{1} K_{2}=L_{1}\left(\frac{\rho}{\Delta \varphi_{\mathrm{e}}}\right)^{2} e^{A^{2} \beta^{2}} /, .
$$

Combined with eqs.(3) and ( 7$), K_{2}$ becomes

$$
K_{2}=e^{\pi Q^{2} / 4\left(1-Q^{2}\right)}
$$

where $Q$, given by Wu et al. $(1985,1986)$, is defined as $Q=\beta / \rho$. When $Q=0$ and $\rho=\Delta \varphi_{\mathrm{e}}$, the

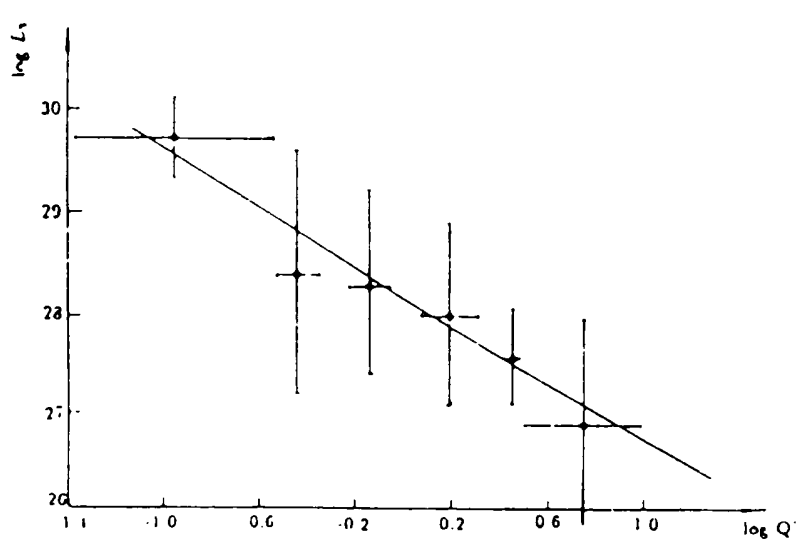

Figure $4 \log L_{3}$ vs. $\log Q^{\prime}\left(Q^{\prime}=2 P^{1.1} \dot{P}_{-15}^{-1.4}\right)$; see figure 3 for key.

values of both $K_{1}$ and $K_{2}$ are 1 , and the new formuia reverts to the old [eq.(9)]. It is obvious that the correction factors of $K_{1}$ and $K_{2}$ are necessary. The value of $L_{1}$ is not a lower limit because $K_{1}<1$ when $\rho<\Delta \varphi_{\mathrm{e}} . K_{2}$ is a function of $Q$ only. In the case of high $Q, K_{2}$ is very large. In the case of lower $Q$ pulsars, such as most pulsars with double or triple profiles, $K_{2} \approx 1$.

\section{Discussion: radio luminosity law}

Studies by Taylor and Stinebring (1986) and Stollman (1987) pointed out that $L$ cannot be described by one relation over the entire range of $P$ and $\dot{P}$. These results are contrary to other previously suggested luminosity laws.

Using the new luminosity formula of eq.(17), our statistical sample contains only 100 pulsars which have good integrated total intensity and polarization profiles. The relations between $\left\langle\log L_{3}\right\rangle$ and $\left\langle\log B P^{-2}\right\rangle$ and between $\left\langle\log L_{3}\right\rangle$ and $\left\langle\log Q^{\prime}\right\rangle$ have been presented. The values of $\left\langle\log L_{3}\right\rangle$ were determined for 6 intervals of $\left\langle\log B P^{-2}\right\rangle$ and $\left\langle\log Q^{\prime}\right\rangle$, ranging from 11.2 to 14.6 of $\left\langle\log B P^{-2}\right\rangle$ and from -1.7 to 1.35 of $\left\langle\log Q^{\prime}\right\rangle$, respectively. The statistical results are shown in figure 3 and figure 4.

There is a close correlation between $\left\langle\log L_{3}\right\rangle$ and $\left\langle\log B P^{-2}\right\rangle(R=0.99) .\left\langle\log L_{3}\right\rangle$ increases steeply with increasing $\left\langle\log B P^{-2}\right\rangle$ over the entire range of $\left\langle\log B P^{-2}\right\rangle$. The regression equation is

$$
\begin{gathered}
\log L_{3}=14.7+1.07 \log B P^{-2} \\
L_{3} \propto\left(B P^{-2}\right)^{1.07} \propto \dot{P}^{0.54} P^{-1.60} .
\end{gathered}
$$

The correlation coefficent between $\left\langle\log L_{3}\right\rangle$ and $\left\langle\log Q^{\prime}\right\rangle$ is high also $(R=-0.96) . L_{3}$ decays steeply 
as $Q^{\prime}$ in the whole range of $Q^{\prime}$. The regression equation is

$$
\begin{gathered}
\log L_{3}=28.2-1.4 \log Q^{\prime} \\
L_{3} \propto\left(Q^{\prime}\right)^{-1.4} \propto \dot{P}^{0.56} P^{-1.54} .
\end{gathered}
$$

These two results shown in eq.(20) and eq.(22) are very similar. Because of $\log B P^{-2} \propto \log \dot{E}_{\text {rot }}, L_{3}$ is best described also by

$$
L_{3} \propto\left(\dot{E}_{\mathrm{rot}}\right)^{0.54}
$$

where the $\dot{E}_{\text {rot }}$ is the loss rate of rotation energy. A natural explanation for this is that the radio luminosity increases with the increasing of loss rate of rotation energy.

\section{Conclusion}

In this paper we have shown that a modification of the radio luminosity formula is necessary and possible. We present a new radio luminosity formula of pulsars based on the geometrical relations of the polar cap model, the statistical result that the intensity distribution of the emission component is Gaussian, and the relation between $\rho$ and $P$ is $\rho=6.5 P^{-1 / 3}$. The large data base of mean pulse intensity and polarization profiles permits us to calculate the new values of radio luminosity. There are 100 pulsars with known new values of radio luminosity.

The radio luminosities (new values) can be described by one relation over the whole range of the period and period derivatives. The results are contrary to the recently suggested luminosity laws given by Taylor et al. (1986) and Stollman (1987). It is reasonable that pulsars are injected into the observable population with longer periods than usually assumed, as suggested by Vivekanand and Narayan (1981).

Acknowledgment: This work was supported by the Natural Science Foundation of China. 